\title{
Antimicrobial Resistance and Molecular Characterization of Gene Cassettes from Class 1 Integrons in Pseudomonas aeruginosa Strains
}

\author{
Mi Liu, Jie Ma, Wei Jia, and Wanxiang Li
}

We investigated the antibiotic-resistance phenotypes and molecularly characterized class 1 integron gene cassettes from 113 Pseudomonas aeruginosa isolates from patients. Primers specific for the class 1 integron integrase (intII) gene were used to screen for these integrons using polymerase chain reactions (PCRs). The variable regions of the integrons were PCR-amplified and sequenced. Sputum was the most common specimen $(69.9 \%$; 79/113) followed by aseptic sites $(21.2 \% ; 24 / 113)$. Of the 113 isolates with phenotypic resistance to the tested antimicrobials, the highest resistances were to ciprofloxacin (CIP) (26.55\%), imipenem (IPM) (23.89\%), and meropenem (MEM) (23\%). Carbapenem-sensitive $P$. aeruginosa (CS-PA) isolates displayed 23 patterns, and the predominant multidrug resistance phenotype was CIP-levofloxacin $(7.23 \%, 6 / 83)$. Carbapenem-resistant $P$. aeruginosa (CR-PA) isolates displayed 12 patterns, and the predominant multidrug resistance phenotype was IPM-MEM $(23.33 \%, 7 / 30)$. Class 1 integrons were detected in $14(12.4 \%, 14 / 113)$ isolates, 7.22\% (6/83) in CS-PA isolates, and 26.67\% (8/30) in CRPA isolates. Six gene cassette arrays were detected, the most prevalent being aacA4-blaOXA101-aadA5 in five isolates $(4.4 \%, 5 / 113)$. Seventeen gene cassettes were detected. The most prevalent antibiotic-resistance gene cassettes were aacA4 $(6.2 \%, 7 / 113), b_{\text {OXA-1 }}$, and $b l a_{O X A-10 I}$. Extended-spectrum $\beta$-lactamase resistance genes were detected. Some of the genes carried were similar to those in other species, but some had shared characteristics among the $P$. aeruginosa isolates. Long-standing drug resistance genes appeared to be under elimination in $P$. aeruginosa, whereas integrons conferring resistance to commonly used clinical drugs such as $\beta$-lactamases, fluoroquinolones, and even carbapenems, as well as some other gene elements, were found to be newly integrated.

Keywords: molecular characterization, antibiotics resistance, class 1 integrons, Pseudomonas aeruginosa

\section{Introduction}

W IDESPREAD AND LONG-TERM use of antibiotics may be responsible for the emergence of antibiotic-resistant strains in several bacterial species. ${ }^{1-5}$ Recent surveillance studies have shown that such strains are markedly resistant to the main antibiotic types and have transformed into the multidrug-resistant (MDR) strains that are nonsusceptible to $\geq 3$ antimicrobial categories. ${ }^{6,7}$

Mobile genetic elements such as plasmids, transposons, and integrons play an important role in increasing antibiotic resistance. ${ }^{6,8,9}$ Class 1 integrons can capture exogenous gene cassettes, thereby ensuring the expression of the genes within these cassettes; hence, they play important roles in the horizontal dissemination of antibiotic resistance genes among bacteria. $^{1-5,10,11}$
Currently, more than 130 different gene cassettes have been found in class 1 integrons, most of which are the antibiotic-resistance gene cassettes encoding proteins with resistance to all main antibiotic classes. ${ }^{12,13}$ These include the quaternary ammonium-compound family, erythromycin, aminoglycosides, sulfonamides, quinolones, chlorampheni$\mathrm{col}$, fosfomycin, trimethoprim, $\beta$-lactamases, and other clinically relevant antibiotics. ${ }^{8,10,14}$

Pseudomonas aeruginosa, an important Pseudomonas genus member, is noted for its ability to cause severe hospitalacquired bloodstream infections, pneumonia, surgical-site infections, and urinary tract infections in intensive care units ${ }^{8,15,16}$ with high morbidity and mortality. ${ }^{17-19}$ Studies have shown that the mortality rate for mechanical ventilation complicated by $P$. aeruginosa pneumonia is $\sim 40 \%$. This pathogen is also the main cause of respiratory tract

Department of Clinical Laboratory, Weifang People's Hospital, Weifang, Shandong, China.

(C) Mi Liu et al., 2020; Published by Mary Ann Liebert, Inc. This Open Access article is distributed under the terms of the Creative Commons Attribution Noncommercial License (http://creativecommons.org/licenses/by-nc/4.0/) which permits any noncommercial use, distribution, and reproduction in any medium, provided the original author(s) and the source are cited. 
infections in patients with cystic fibrosis, as well as a common cause of disease aggravation in patients with advanced chronic obstructive pulmonary disease.

Therefore, $P$. aeruginosa attracts attention from medical staff. ${ }^{8}$ Previously, it was thought that carbapenem antibiotics were the last line of defense against multiple drug-resistant, gram-negative bacterial infections. However, with the inappropriate and overuse of antibiotics, there has been a selection pressure for antibiotic-resistant strains to evolve. P. aeruginosa has a multiple drug resistance phenotype, and even harbors carbapenem resistance. ${ }^{8}$ Many studies have investigated the prevalence of integrons in $P$. aeruginosa, but its drug resistance spectrum is rarely reported. Therefore, comparison of the integrons carried by carbapenem-resistant $P$. aeruginosa (CRPA) strains with carbapenem-sensitive $P$. aeruginosa (CS-PA) strains and characterizing and comparing the gene cassettes from this bacterium are a worthwhile exercise. ${ }^{17}$

Therefore, we investigated drug resistance in $P$. aeruginosa isolated from the clinic, the carriage of class I integrons among CR-PA strains and CS-PA strains, and the characteristics of the variable regions of class I integrons from this bacterium.

\section{Materials and Methods}

\section{Bacterial strains}

Altogether, $113 P$. aeruginosa strains were isolated from patients hospitalized in Weifang People's Hospital (Weifang, China). These isolates were collected from September to October 2018. We have received and archived written patient consent from all patients included in this study, and the approval by the hospital ethics committee for the use of samples for human patients. Patients needed to have been hospitalized $>3$ days to qualify for specimen collection. There were obvious symptoms of infection in each system. Symptoms of tissue infection were redness, necrosis, or exudation. Symptoms of respiratory tract infection were fever, cough, and imaging findings. Examples of systemic symptoms were shivering, fever, as well as increases in levels of leukocytes, erythrocyte sedimentation rate, and C-reactive protein.

All specimens were collected and transported in aseptic containers. For respiratory specimens, the patient had to gargle before coughing-up sputum. After each sputum sample had been sent to the laboratory, the number of white cells was $>25$ per low magnification, and the number of squamous cells was $<10$ per low magnification, and all were identified by Vitek-2 Compact (BioMérieux, Marcyl'Étoile, France). Escherichia coli DH5 $\alpha$ was from our laboratory. Proteus mirabilis 47437 was gifted by the Clinical Laboratory of Zhejiang Province People's Hospital (Zhejiang, China).

\section{Antimicrobial susceptibility testing}

Several antibiotics were selected as representative drugs. Antimicrobial susceptibility testing on these antibiotics was done using the disk diffusion method according to the Clinical and Laboratory Standards Institute (CLSI) guidelines. ${ }^{20}$ The antibiotics chosen were amikacin (AK), ceftazidime, ciprofloxacin (CIP), levofloxacin (LEV), cefepime, gentamicin, tobramycin (TOB), imipenem (IPM), aztreonam (ATM), cefoperazone/sulbactam (CSL), piperacillin (PRL), piperacillin/tazobactam (TZP), and meropenem (MEM). Isolates shown to be resistant to IPM or MEM were defined as "CR-PA," and those resistant to three or more drugs class were defined as "MDR-PA." P. aeruginosa ATCC 27853 was used as the control for antibiotic resistance.

\section{Polymerase chain reaction assay for screening class 1 integrons}

All strains were streaked onto Luria Bertani (Oxoid, UK) agar plates and incubated overnight at $37^{\circ} \mathrm{C}$. A single clone from each strain was picked and suspended in $50 \mu \mathrm{L}$ of distilled water. After incubation $\left(100^{\circ} \mathrm{C}\right.$ water bath, 10 minutes) and centrifugation $(13,750 \mathrm{~g}, 2$ minutes), the supernatant was used as the polymerase chain reaction (PCR) template for screening of class 1 integrons. Primers intF and $\mathrm{P}_{2} \mathrm{R}$ (Table 1), which target the class 1 integron integrase (intI1), were used. A blank control (distilled water), a negative control (E. coli $\mathrm{DH} 5 \alpha)$, and a positive control $(P$. mirabilis 47437) were included in each run.

\section{Identifying the variable regions of class 1 integrons}

Because boiling method is a rough extraction method, which nucleic acid concentration may decrease over time, this method can be used in the preliminary screening of integron. To ensure the reproducibility and stability of the experimental results, the reagent extraction method should be used in further experiments. Therefore, we adopt the method of extracting DNA by kit to screen the variable region of integron. Total genomic DNA was isolated from the class 1 integron-positive strains using the EZ-10 Spin Column Bacterial Genomic DNA Miniprep Kit (Bio Basic, Canada) according to the supplier's instructions. Table 1 shows the primers used in this study. Primers 5CS and 3CS were used to amplify the variable regions of the class 1 integrons.

For the PCRs, LA Taq DNA polymerase (TaKaRa Biotechnology, Japan) was used according to the manufacturer's instructions. After electrophoresis on a $1 \%$ agarose gel, the PCR products were excised and purified according to the manufacturer's instructions of the EZ-10 Spin Column Gel Extraction Kit (Bio Basic). All amplicons were sequenced by primer walking,

Table 1. PCR Primers Used In This Study

\begin{tabular}{lllc}
\hline Gene & \multicolumn{1}{c}{ Description } & \multicolumn{1}{c}{ Sequence $\left(5^{\prime} \rightarrow 3^{\prime}\right)$} & Reference \\
\hline intF & Screening primer for class 1 integrons & CCAAGCTCTCGGGTAACATC & 5 \\
$P 2 R$ & Screening primer for class 1 integrons & GCCCAGCTTCTGTATGGAAC & 5 \\
$5 C S$ & Screening primer for the variable region of class 1 integrons & GGCATCCAAGCAGCAAG & 5 \\
$3 C S$ & Screening primer for the variable region of class 1 integrons & AAGCAGACTTGACCTGA & 5 \\
\hline
\end{tabular}


starting with 3CS and 5CS. Nucleotide sequences were analyzed and compared using BLAST software.

\section{Statistical analysis}

The data were analyzed using SSPS v17.0 (IBM, Armonk, NY). Comparisons were considered significant if $p<0.05$.

\section{Results}

\section{Clinical samples and bacterial isolation}

Altogether, 113 P. aeruginosa isolates were collected from hospitalized patients. Most specimens were sputum (69.9\%; 79/113), followed by aseptic site specimens $(21.2 \%$; 24/113), blood $(5.3 \% ; 6 / 113)$, and urine $(3.5 \% ; 4 / 113)$. CR-PA and CS-PA were 30 and 83 , respectively.

\section{Resistance phenotypes}

Table 2 shows the antimicrobial resistance phenotypes of the isolates toward 13 antimicrobial agents. Antimicrobial susceptibility testing revealed that of the 113 isolates expressing phenotypic resistance to the tested antimicrobials, the most common resistance was to CIP (26.55\%) followed by IPM (23.89\%) and MEM (23\%); all other antimicrobials had values of $<20 \%$ (Table 2 ). P. aeruginosa multidrugresistant isolates (resistant to at least three antimicrobial classes) were recovered from the two sets of isolates investigated in our study. Isolates with resistance to all the antimicrobials tested occurred only in the CR-PA isolates. The highest prevalence of resistance in the CR-PA isolates was to IPM $(93.33 \%)$ followed by MEM (86.67\%), LEV (56.67\%), and CIP $(53.33 \%)$; all other antimicrobials had values of $<40 \%$. In the CS-PA isolates, the most common resistance was to LEV (16.87\%), followed by CIP (15.66\%); all other antimicrobials had values of $<20 \%$. Comparison of drug resistance in the CS-PA and CR-PA groups revealed that, with the exception of GEN and ATM, the other 11 antimicrobial agents differed, and the differences were significant.

Table 2. Resistance Phenotypes and Comparison of CARbapenem-Resistant PSEUdomonas AERUGINOSA With CARBAPENEM-SENSITIVE $P$. AERUGINOSA

\begin{tabular}{lcccc}
\hline & CR-PA & CS-PA & Total $(\%)$ & $\mathrm{p}$ \\
\hline AK & 23.33 & 0 & 7.07 & 0.000 \\
ATM & 26.67 & 14.46 & 17.70 & 0.222 \\
CAZ & 36.67 & 3.61 & 12.39 & 0.000 \\
CIP & 53.33 & 15.66 & 26.55 & 0.000 \\
CSL & 36.67 & 6.02 & 15.04 & 0.000 \\
FEP & 36.67 & 0 & 9.73 & 0.000 \\
GEN & 16.67 & 6.02 & 9.73. & 0.079 \\
IPM & 93.33 & 0 & 23.89 & - \\
LEV & 56.67 & 16.87 & 27.4 & 0.000 \\
MEM & 86.67 & 0 & 23.0 & - \\
PIP & 36.67 & 13.25 & 19.46 & 0.006 \\
TOB & 30.00 & 4.81 & 12.39 & 0.001 \\
TZP & 30.00 & 6.02 & 12.39 & 0.001 \\
\hline
\end{tabular}

AK, amikacin; ATM, aztreonam; CAZ, ceftazidime; CIP, ciprofloxacin; CR-PA, carbapenem-resistant $P$. aeruginosa; CSL, cefoperazone/sulbactam; CS-PA, carbapenem-sensitive P. aeruginosa; FEP, cefepime; GEN, gentamicin; IPM, imipenem; LEV, levofloxacin; MEM, meropenem; PRL, piperacillin; TOB, tobramycin; TZP, piperacillin/tazobactam.

\section{Resistance patterns}

The strains were divided into two groups based on their carbapenem resistance levels (CR-PA, 30 strains; CS-PA, 83 strains) and the definition rules. The total number of multidrug-resistant $P$. aeruginosa (MDR-PA) isolates in the CS-PA group was $16.87 \%$ (14/83), whereas that of CR-PA was $40 \%(12 / 30)$ (Table 3$)$. The CS-PA isolates displayed 23 patterns, the predominant multidrug resistance pattern of which was CIP-LEV (7.23\%, 6/83) followed by CIP-LEVIPM-MEM (3.61\%, 3/83). ATM and CIP-LEV-IPM patterns were displayed by two strains. Only one strain exhibited another resistance pattern. Twelve patterns were seen for the CR-PA isolates, and the predominant multidrug resistance pattern was IPM-MEM $(23.33 \%, 7 / 30)$, followed by AKATM-CAZ-CIP-CSL-FEP-IPM-LEV-MEN-PRL-TOB-TZP (10\%, 3/30). Sixty $P$. aeruginosa isolates displayed sensitivity to all 13 antimicrobial agents.

\section{Prevalence of class 1 integrons and amplification products from the variable regions}

Class 1 integrons were detected in 14 (12.4\%, 14/113) isolates (Table 3$), 7.22 \%(6 / 83)$ in the CS-PA isolates and $26.67 \%(8 / 30)$ in the CR-PA isolates. The variable regions of the class 1 integrons were amplified and analyzed successfully for 10 intI1-positive isolates, but these regions failed to amplify in four intI1-positive isolates. In the remaining 10 intIl-positive isolates, only partial variable region sequences were amplified and sequenced.

Six gene cassette arrays were detected (Table 3), the most prevalent of which were aacA4-blaOXA101-aadA5 (detected in five strains), whereas qnrvc-gcul65-arr2-dfrA22e, aacA4gcu35-blaOXA-1-catB3, aadB-aac(6')-1I-pse-1, dhfr2-aacA4aadA1-tna, and AadA22 were detected in 2, 2, 1, 1, and 1 isolates, respectively. aacA4-blaOXA101-aadA5, aacA4gcu35-blaOXA-1-catB3, and qnrvc-gcu165-arr2-dfrA22e gene cassette arrays were detected in CR-PA at 5/30, 2/30, and 2/30, respectively, whereas aadB-aac $\left(6^{\prime}\right)$-1I-pse-1, dhfr2-aacA4aadA1-tna, and AadA22 were detected in CS-PA in 1, 1, and 1 isolates, respectively. Strain numbers 39 and 70 both contained two gene cassette arrays (qnrvc-gcu165-arr2-dfrA22e and aacA4-gcu35-blaOXA-1-catB3).

Seventeen gene cassettes were detected, including blaOXA-1/101, pse-1, aacA4, aac6-II, aadA1/A5//B, AadA22, arr2, catB3, dfrA22e, dhfr2, tha, qnrvc, and gcu35/ 165 (Table 3 ). The most prevalent antibiotic resistance gene cassettes were aacA4 (7/113), which confers resistance to aminoglycosides, aadA5 (5/113), which confers resistance to streptomycin and spectinomycin, and blaOXA-101 (5/ $113)$, which confers resistance to $\beta$-lactamases. The structural characteristics and translation directions of the variable region gene cassettes are shown in Fig. 1.

\section{Comparison between integron-positive and integron-negative isolates}

Of the 14 integron-positive isolates, $>70 \%$ were resistant to CIP, LEV, and TOB, and $>50 \%$ were resistant to the other drugs. In the integron-negative isolates, only resistance to IPM and MEM was close to $20 \%$, whereas resistance to the other drugs was $<20 \%$ or close to 0 . Comparison of the drug sensitivity of the two groups revealed that the drug 
Table 3. Antimicrobial Resistance Phenotypes and Molecular Characterization of Gene Cassettes in Class 1 Integrons

\begin{tabular}{|c|c|c|c|c|c|}
\hline Sample no. & Resistance pattern & Int & Length & Gene cassettes & $\begin{array}{c}\text { GenBank } \\
\text { accession no. }\end{array}$ \\
\hline $\begin{array}{l}1,2,4,8,9,15,17,20,22,26, \\
27,29,30,31,34,38,38,44, \\
45,47,49,50,52,53,55,56 \\
57,60,62,63,64,65,66,67 \\
\text { 68, 69, 73, 74, 75, 76, 77, 78, } \\
79,80,82,84,89,93,95,96 \\
98,102,106,107,109,110, \\
112,114,115,116\end{array}$ & Sensitive to all drugs & - & - & - & \\
\hline \multicolumn{6}{|l|}{ CS-PA } \\
\hline 3,83 & ATM & - & - & - & \\
\hline 117 & PRL & - & - & - & \\
\hline $25,51,92,94,99,105$ & CIP, LEV & - & - & - & \\
\hline 85 & ATM, PRL & - & - & - & \\
\hline 13 & LEV, PRL & - & - & - & \\
\hline 41 & ATM, CIP, LEV & - & - & - & \\
\hline 100 & ATM, CIP, LEV & + & - & - & \\
\hline 43 & CAZ, PRL, TZP & - & - & - & \\
\hline 5,88 & CIP, LEV, IPM & - & - & - & \\
\hline 61 & CIP, LEV, PRL & - & - & - & \\
\hline 104 & CIP, LEV, GEN & + & - & - & \\
\hline 35 & ATM, CSL, PRL & + & $1.1 \mathrm{k}$ & AadA22 & $\begin{array}{c}\mathrm{CP} 041394.1 \\
99.48 \%\end{array}$ \\
\hline 23 & GEN, PRL, TOB, TZP & + & $2.1 \mathrm{k}$ & $\begin{array}{l}a a d B-a a c\left(6^{\prime}\right)- \\
\text { 1I-pse-1 }\end{array}$ & $\begin{array}{l}D Q 266447.1 \\
97.6\end{array}$ \\
\hline 32 & ATM, CSL, PRL, TZP & - & - & - & \\
\hline $6,11,19$ & CIP, LEV, IPM, MEM & & - & - & \\
\hline 48 & ATM, CIP, LEV, MEM & - & - & - & \\
\hline 16 & CSL, LEV, IPM, MEM & - & - & - & \\
\hline 87 & ATM, CIP, GEN, LEV, TOB & - & - & - & \\
\hline 108 & ATM, CAZ, CSL, PRL, TOB & - & - & - & \\
\hline 18 & CAZ, CIP, FEP, GEN, LEV, TOB & + & - & - & \\
\hline 111 & AK, ATM, CIP, CSL, GEN, TOB & + & $3.2 \mathrm{k}$ & $\begin{array}{l}\text { dhfr2-aacA4- } \\
\text { aadA1-tna }\end{array}$ & \\
\hline 97 & $\begin{array}{l}\text { ATM, CIP, CSL, GEN, LEV, PRL, } \\
\text { TOB }\end{array}$ & - & - & & \\
\hline 42 & $\begin{array}{l}\text { ATM, CAZ, CIP, CSL, GEN, LEV, } \\
\text { PRL, TOB, TZP }\end{array}$ & - & - & - & \\
\hline \multicolumn{6}{|c|}{ 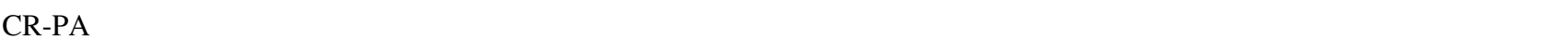 } \\
\hline 21 & IPM, MEM, LEV & - & - & - & \\
\hline 14 & IPM, MEM, PRL & - & - & - & \\
\hline $7,10,12,40,59,81,113$ & IPM, MEM & - & - & - & \\
\hline 72 & CAZ, IPM, MEM, PRL & - & - & - & \\
\hline 103 & CIP, FEP, LEV, IPM, MEM & - & - & - & \\
\hline 28 & $\begin{array}{l}\text { ATM, CAZ, CSL, FEP, IPM, MEM, } \\
\text { PRL }\end{array}$ & - & - & - & \\
\hline 33 & $\begin{array}{l}\text { ATM, CAZ, CSL, IPM, MEM, PRL, } \\
\text { TZP }\end{array}$ & - & - & - & \\
\hline 54 & $\begin{array}{l}\text { CIP, CSL, FEP, GEN, IPM, LEV, } \\
\text { MEN, PRL, TOB, TZP }\end{array}$ & + & - & - & \\
\hline 71 & $\begin{array}{l}\text { AK, ATM, CAZ, CIP, CSL, FEP, } \\
\text { IPM, MEN, PRL, TOB, TZP }\end{array}$ & + & $2.1 \mathrm{k}$ & $\begin{array}{l}\text { aacA4-blaOXA101- } \\
\text { aadA5 }\end{array}$ & \\
\hline $36,46,101$ & $\begin{array}{l}\text { AK, ATM, CAZ, CIP, CSL, FEP, } \\
\text { IPM, LEV, MEN, PRL, TOB, TZP }\end{array}$ & + & $2.1 \mathrm{k}$ & $\begin{array}{l}\text { aacA4-blaOXA101- } \\
\text { aadA5 }\end{array}$ & \\
\hline 39,70 & $\begin{array}{l}\text { AK, CAZ, CIP, CSL, FEP, GEN, } \\
\text { IPM, LEV, MEN, PRL, TOB, TZP }\end{array}$ & + & $2.2 \mathrm{k}+2.8 \mathrm{k}$ & $\begin{array}{c}\text { qnrvc-gcu165- } \\
\text { arr2-dfrA22e } \\
\text { aacA4-gcu35- } \\
\text { blaOXA-1-catB3 }\end{array}$ & $\begin{array}{l}\text { KU984332 } \\
\quad \text { KU984333 }\end{array}$ \\
\hline 24 & $\begin{array}{l}\text { AK, ATM, CAZ, CIP, CSL, FEP, } \\
\text { GEN, IPM, LEV, MEN, PRL, } \\
\text { TOB, TZP }\end{array}$ & + & $2.1 \mathrm{k}$ & $\begin{array}{l}\text { aacA4-blaOXA101- } \\
\text { aadA5 }\end{array}$ & $\begin{array}{l}\text { KM111259.1 } \\
\quad 99.35 \%\end{array}$ \\
\hline
\end{tabular}

+ , presence of the PCR product; -, absence of the PCR product.

ATM, aztreonam (30 mg); IPM, imipenem (10 mg). 
FIG. 1. Genetic environment of class 1 integron variable regions. (A) Isolate 35. (B) Isolate 23. (C) Isolates 24, 36, 46, 71, and 101. (D1, D2) Isolates 39 and 70.

(E) Isolate 111

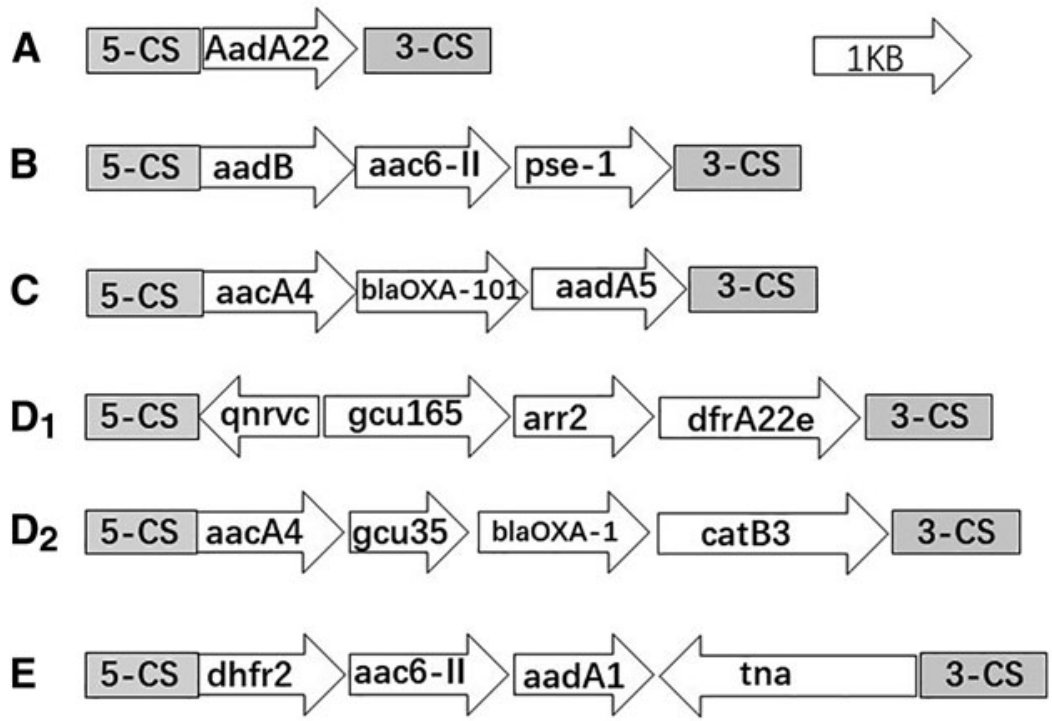

resistance rates for the 13 antimicrobial agents in the integron-positive isolates were higher than those of the integron-negative isolates, and the difference was significant (Table 4).

\section{Discussion}

In recent years, the emergence and spread of MDR bacteria have brought great challenges to the clinical treatment of bacterial infections. $P$. aeruginosa, a clinically important Pseudomonas species, causes nosocomial infections worldwide. During transmission of bacterial drug resistance, the movement of genes and other DNA sequences (e.g., drug resistance plasmids, transposons, insertion sequences, and integrons, among others) plays an important role. Increased antibiotic resistance in $P$. aeruginosa isolates can be attributed to their ability to obtain resistance by the movement of genetic elements. Because of the role played by class 1 integrons in the rapid dissemination of multiple genes encoding antibiotic resistance, ${ }^{6}$ we analyzed the prevalence of class 1 integrons in $P$. aeruginosa.

Table 4. Comparison Between Integron-Positive AND INTEGRON-NEGATIVE ISOLATES

\begin{tabular}{lccc}
\hline & int $+(\%)$ & int $-(\%)$ & $\mathrm{p}$ \\
\hline AK & 50.00 & 0.00 & 0.000 \\
ATM & 57.14 & 12.12 & 0.000 \\
CAZ & 57.14 & 6.06 & 0.000 \\
CIP & 78.57 & 18.18 & 0.000 \\
CSL & 64.29 & 7.07 & 0.000 \\
FEP & 64.29 & 2.02 & 0.000 \\
GEN & 50.00 & 3.03 & 0.000 \\
IPM & 57.14 & 20.20 & 0.003 \\
LEV & 71.43 & 21.21 & 0.000 \\
MEM & 57.14 & 18.18 & 0.001 \\
PIP & 64.29 & 13.13 & 0.000 \\
TOB & 71.43 & 3.03 & 0.000 \\
TZP & 64.29 & 5.05 & 0.000 \\
\hline
\end{tabular}

int+: integron-positive isolates; int-: integron negative isolates.
Altogether, $113 P$. aeruginosa isolates were collected, primarily from sputum $(69.9 \% ; 79 / 113)$, aseptic site specimens $(21.2 \% ; 24 / 113)$, blood $(5.3 \% ; 6 / 113)$, and urine $(3.5 \% ; 4 / 113)$.

The isolates from our study were CR-PA and CS-PA types, comprising 30 and 83, respectively. Excluding IPM and MEM, of the 11 other drugs, only GEN did not show a significant difference in resistance. Resistance in the CR-PA group was greater than that of the CS-PA group, and the difference was significant, a finding also shown by $\mathrm{Gu} \mathrm{B}^{21}$ and colleagues. This finding suggests that new drug-resistant genes are captured continuously by $P$. aeruginosa under the selection pressure of an antibacterial agent, which enables $P$. aeruginosa to survive if various medicines are administered. In addition, based on the integron carrier, the collected strains were divided into integron-positive and integron-negative groups, from which drug resistance was compared. The results showed more integron-positive groups than integron-negative groups in all 13 commonly used clinical drugs, and the difference was significant. This result is similar to that of other scholars. $5,21-23$

Class 1 integrons were detected in $14(12.4 \%, 14 / 113)$ isolates, a similar finding to other research from China. 5,21-23 However, this result is quite different from those of studies in which the determinants of drug resistance related to integrons were found in the isolates from several $P$. aeruginosa outbreaks. ${ }^{6,24,25}$ Studies have shown that almost half of all drug-resistant $P$. aeruginosa strains carry integrons. ${ }^{6,26}$ It has been suggested that the homozygous carriage rate for $P$. aeruginosa in China is lower than that in other countries, and the results of our study are in accordance with data from China. ${ }^{5,21}$

We detected six gene clusters containing 17 gene cassettes in the variable regions of the isolates. These gene cassettes contain not only aminoglycoside and trimethoprim drug-resistant genes (the antibiotics for which were used commonly in China in the early to mid-20th century) but also genes encoding resistance to $\beta$-lactamases, fluoroquinolones, and even carbapenem (the antibiotics for which are used widely at present). Previously, these genes were underdetected or detected mainly in other species, 
whereas in $P$. aeruginosa, they were relatively rare. Furthermore, a transposition enzyme, genome gene cassettes ( gcu35/165), and various other integrons were detected in our study, and this was relatively rare in the variable regions of integrons in the past. The specific functions of the integrons in these gene cassettes are obscure and must be studied further.

Novel genes conferring antibiotic resistance, such as qnVC6, have been reported in different species. $6,27-29$ blaOXA-01/101, which mainly mediates drug resistance in Acinetobacter baumannii, also had a high detection rate in our study. In addition, the diversity of gene cassettes carried by class 1 integrons is broadening, ${ }^{6,30}$ and aad cassettes (which confer resistance to aminoglycosides) were detected herein with a similarly high detection rate as that reported previously. However, gene cassettes encoding resistance to trimethoprim (dfrA) were found less frequently in our study. Previously, it was considered that this gene is highly stable in Enterobacteriaceaeintegrated zygotes, with high detection rates in most strains. That the detection rate of dfrA in the present study was low may reflect that $P$. aeruginosa has a strong ability to respond quickly to the selection pressure of antibiotics. ${ }^{31-33}$

Although the frequency of isolates resistant to $\beta$-lactamases was high, the blaOXA-1 gene cassette was found in only one commensal isolate, suggesting that the genes encoding $\beta$ lactamases are mostly located outside of integrons, which was also observed by Mokracka et al. ${ }^{34,35}$ The extended-spectrum $\beta$ lactamase resistance genes-blaOXA-1 and blaOXA-101were also found in the present study. These genes can confer resistance to cefepime and IPM, the most commonly used antibiotics nowadays. However, these gene cassettes were found in only two intI1-positive isolates. Possibly the selection pressure of these antibiotics lacks strength or insufficient time has passed to select for a higher prevalence of these gene cassettes. $5,6,21,31-35$

\section{Conclusions}

Our data suggest that $P$. aeruginosa is found commonly in clinical respiratory tract infections and aseptic site infections, and drug resistance in it has been increasing considerably in recent years. The integron carriage rate of $P$. aeruginosa and the characteristics of the gene cassettes it carries suggest that there are similarities and differences in the genes it carries compared with other species. Stably acquired drug resistance genes are being eliminated in $P$. aeruginosa.

Concurrently, genetic resistance elements against commonly used clinical drugs (e.g., $\beta$-lactamases, fluoroquinolones, and even carbapenems), as well as some other gene elements, are newly integrated. The variable regions of the integrons showed that the gene clusters we identified were, in general, large, and there were many gene cassette types, both of which play a very important role in drug resistance in $P$. aeruginosa. The continuous evolution of drug resistance in $P$. aeruginosa brings great challenges to its clinical treatment. Therefore, curbing drug resistance and the spread of $P$. aeruginosa infections are important goals.

\section{Authors' Contributions}

W.L. designed the study. W.L., M.L., and J.M. conducted the study, collected the data, and prepared the article. W.J. provided advice and edited the article. All authors approved the final version of the article.

\section{Acknowledgments}

DNA sequencing and primer synthesis were carried out by Sangon Biotechnology Co., Ltd. (Shanghai, China). We thank Sandra Cheesman, PhD, from Liwen Bianji, Edanz Group China, for editing the English text of a draft of this article. We have received and archived written patient consent from all patients included in this study, and the approval by the hospital ethics committee for the use of samples for human patients.

\section{Disclosure Statement}

No competing financial interests exist.

\section{Funding Information}

The present study was supported by the fund from the Health and Family Planning Commission of Weifang (wfwsjs_2018_110) and research grants from the Health Commission of Shandong Province (2018WS078).

\section{References}

1. Hall, R.M., and H.W. Stokes. 1993. Integrons: novel DNA elements which capture genes by site-specific recombination. Genetica 90:115-132.

2. Collis, C.M., and R.M. Hall. 1995. Expression of antibiotic resistance genes in the integrated cassettes of integrons. Antimicrob. Agents Chemother. 39:155-162.

3. Hall, R.M., and C.M. Collis. 1998. Antibiotic resistance in Gram-negative bacteria: the role of gene cassettes and integrons. Drug Resist. Updat. 1:109-119.

4. Mazel, D. 2006. Integrons: agents of bacterial evolution. Nat. Rev. Microbiol. 4:608-620.

5. Wei, Q., X. Jiang, M. Li, G. Li, Q. Hu, H. Lu, G. Chen, Y. Zhou, and Y. Lu. 2013. Diversity of gene cassette promoter variants of class 1 integrons in uropathogenic Escherichia coli. Curr. Microbiol. 67:543-549.

6. Novovic, K.D., M.J. Malesevic, B.V. Filipic, N.L. Mirkovic, M.S. Miljkovic, M.O. Kojic, and B.U. Jovčić. 2019. PsrA regulator connects cell physiology and class 1 integron integrase gene expression through the regulation of lexA gene expression in Pseudomonas spp. Curr. Microbiol. 76:320-328.

7. Magiorakos, A.P., A. Srinivasan, R.B. Carey, Y. Carmeli, M.E. Falagas, C.G. Giske, S. Harbarth, J.F. Hindler, G. Kahlmeter, B. Olsson-Liljequist, D.L. Paterson, L.B. Rice, J. Stelling, M.J. Struelens, A. Vatopoulos, J.T. Weber, and D.L. Monnet. 2012. Multidrug-resistant, extensively drugresistant and pandrug-resistant bacteria: an international expert proposal for interim standard definitions for acquired resistance. Clin. Microbiol. Infect. 18:268-281.

8. Kung, V.L., E.A. Ozer, and A.R. Hauser. 2010. The accessory genome of Pseudomonas aeruginosa. Microbiol. Mol. Biol. Rev. 74:621-641.

9. Livermore, D.M. 2002. Multiple mechanisms of antimicrobial resistance in Pseudomonas aeruginosa: our worst nightmare? Clin. Infect. Dis. 34:634-640.

10. Stokes, H.W., and R.M. Hall. 1989. A novel family of potentially mobile DNA elements encoding site-specific gene-integration functions: integrons. Mol. Microbiol. 3: 1669-1683.

11. Recchia, G.D., and R.M. Hall. 1995. Gene cassettes: a new class of mobile element. Microbiology 141:3015-3027. 
12. Partridge, S.R., G. Tsafnat, E. Coiera, and J. Iredell. 2009. Gene cassettes and cassette arrays in mobile resistance integrons. FEMS Microbiol. Rev. 33:757-784.

13. Cambray, G., A. Guerout, and D. Mazel. 2010. Integron. Annu. Rev. Genet. 44:141-166.

14. Deng, Y., J. Liu, B. Peters, D. Chen, G. Yu, Z. Xu, and M. Shirtliff. 2015. Antimicrobial resistance investigation on Staphylococcus strains in a local hospital in Southern China, 2001-2010. Microb. Drug Resist. 21:102-104.

15. Carmeli, Y., N. Troillet, A.W. Karchmer, and M.H. Samore. 1999. Health and economic outcomes of antibiotic resistance in Pseudomonas aeruginosa. Arch. Intern. Med. 159:1127-1132.

16. Jarvis, W.R. 2003. Epidemiology and control of Pseudomonas aeruginosa infections in the intensive care unit. In A.R. Hauser and J. Rello (ed.), Severe infections caused by Pseudomonas aeruginosa. Kluwer Academic Publishers, Boston, MA, pp. 153-168.

17. Zhang, R., Z. Liu, J. Li, L. Lei, W. Yin, M. Li, C. Wu, T.R. Walsh, Y. Wang, S. Wang, and Y. Wu. 2017. Presence of VIM-positive Pseudomonas species in chickens and their surrounding environment. Antimicrob. Agents Chemother. 27;61:e00167-17.

18. Wang, Y., R. Zhang, J. Li, Z. Wu, W. Yin, S. Schwarz, M.J. Tyrrell, Y. Zheng, S. Wang, Z. Shen, Z. Liu, J. Liu, L. Lei, M. Li, Q. Zhang, C. Wu, Q. Zhang, Y. Wu, T. Walsh, and J. Shen. 2017. Comprehensive resistome analysis reveals the prevalence of NDM and MCR-1 in Chinese poultry production. Nat. Microbiol. 2:16260.

19. Nordmann, P., M. Gniadkowski, C.G. Giske, L. Poirel, N. Woodford, V. Miriagou; and European Network on Carbapenemases. 2012. Identification and screening of carbapenemase-producing Enterobacteriaceae. Clin. Microbiol. Infect. 18:432-438.

20. Clinical and Laboratory Standards Institute. 2018. Performance Standards for Antimicrobial Susceptibility Testing. 28th ed. CLSI, Wayne, PA. CLSI supplement M100.

21. Gu, B., M. Tong, W. Zhao, G. Liu, M. Ning, S. Pan, and W. Zhao. 2007. Prevalence and characterization of class I integrons among Pseudomonas aeruginosa and Acinetobacter baumannii isolates from patients in Nanjing, China. J. Clin. Microbiol. 45:241-243.

22. Lee, Y.L., M.C. Lu, P.L. Shao, P.L. Lu, Y.H. Chen, S.H. Cheng, W.C. Ko, C.Y. Lin, T.S. Wu, M.Y. Yen, L.S. Wang, C.P. Liu, W.S. Lee, Z.Y. Shi, Y.S. Chen, F.D. Wang, S.H. Tseng, C.N. Lin, Y.H. Chen, W.H. Sheng, C.M. Lee, M.H. Liao, and P.R. Hsueh. 2019. Nationwide surveillance of antimicrobial resistance among clinically important Gram-negative bacteria, with an emphasis on carbapenems and colistin: results from the Surveillance of Multicenter Antimicrobial Resistance in Taiwan (SMART) in 2018. Int. J. Antimicrob. Agents. 54:318-328.

23. Ayoub Moubareck, C., D. Hammoudi Halat, C. Akkawi, A. Nabi, M.A. AlSharhan, Z.O. AlDeesi, C.C. Peters, H. Celiloglu, and D. Karam Sarkis. 2019. Role of outer membrane permeability, efflux mechanism, and carbapenemases in carbapenem-nonsusceptible Pseudomonas aeruginosa from Dubai hospitals: results of the first cross-sectional survey. Int. J. Infect. Dis. 84:143-150.

24. Kouda, S., M. Ohara, M. Onodera, Y. Fujiue, M. Sasaki, T. Kohara, S. Kashiyama, S. Hayashida, T. Harino, T. Tsuji, H. Itaha, N. Gotoh, A. Matsubara, T. Usui, and M. Sugai. 2009. Increased prevalence and clonal dissemination of multidrug-resistant Pseudomonas aeruginosa with the
blaIMP-1 gene cassette in Hiroshima. J. Antimicrob. Chemother. 64:46-51.

25. Tsakris, A., A. Poulou, I. Kristo, T. Pittaras, N. Spanakis, S. Pournaras, and F. Markou. 2009. Large dissemination of VIM-2-metallo $\beta$ Pseudomonas aeruginosa strains causing health care-associated community-onset infections. J. Clin. Microbiol. 47:3524-3529.

26. Xu, Z., L. Li, M.E. Shirtliff, M.J. Alam, S. Yamasaki, and L. Shi. 2009. Occurrence and characteristics of class 1 and 2 integrons in Pseudomonas aeruginosa isolates from patients in southern China. J. Clin. Microbiol. 47:230-234.

27. Liu, J., L. Yang, D. Chen, B.M. Peters, L. Li, B. Li, Z. Xu, and M.E. Shirtliff. 2018. Complete sequence of pBM413, a novel multidrug resistance megaplasmid carrying qnrVC6 and blaIMP-45 from Pseudomonas aeruginosa. Int. J. Antimicrob. Agents 51:145-150.

28. Liu, J., L. Yang, L. Li, B. Li, D. Chen, and Z. Xu. 2018. Comparative genomic analyses of two novel qnrVC6 carrying multidrug-resistant Pseudomonas spp. strains. Microb. Pathog. 123:269-274.

29. Liu, J., L. Li, B.M. Peters, B. Li, D. Chen, Z. Xu, and M.E. Shirtliff. 2018. Complete genomic analysis of multidrugresistance Pseudomonas aeruginosa Guangzhou-Pae617, the host of megaplasmid pBM413. Microb. Pathog. 117:265-269.

30. Yu, G., W. Wen, B. Peters, J. Liu, C. Ye, Y. Che, J. Liu, K. Cao, Z. Xu, and M.E. Shirtliff. 2016. First report of novel genetic array aacA4blaIMP-25-oxa30-catB3 and identification of novel metallo- $\beta$ lactamase gene blaIMP25: a retrospective study of antibiotic resistance surveillance on Psuedomonas aeruginosa in Guangzhou of South China, 2003-2007. Microb. Pathog. 95:62-67.

31. Zeighami, H., F. Haghi, N. Masumian, F. Hemmati, A. Samei, and G. Naderi. 2015. Distribution of integrons and gene cassettes among uropathogenic and diarrheagenic Escherichia coli isolates in Iran. Microb. Drug Resist. 21:435-440.

32. Su, J., L. Shi, L. Yang, Z. Xiao, X. Li, and S. Yamasaki. 2006. Analysis of integrons in clinical isolates of Escherichia coli in China during the last six years. FEMS Microbiol. Lett. 254:75-80.

33. Yan, H., L. Li, M. Zong, M.J. Alam, and S. Shinoda. 2010. Occurrence and characteristics of class I and II integrons in clinical bacterial isolates from patients in south China. J. Health Sci. 56:442-450.

34. Oliveira-Pinto, C., C. Diamantino, P.L. Oliveira, M.P. Reis, P.S. Costa, M.C. Paiva, R.M.D. Nardi, P.P. Magalhães, E. Chartone-Souza, and A.M.A. Nascimento. 2017. Occurrence and characterization of class 1 integrons in Escherichia coli from healthy individuals and those with urinary infection. J. Med. Microbiol. 66:577-583.

35. Mokracka, J., R. Koczura, and A. Kaznowski. 2012. Transferable integrons of Gram-negative bacteria isolated from the gut of a wild boar in the buffer zone of a national park. Ann. Microbiol. 62:877-880.

Address correspondence to: Wanxiang Li, MD

Department of Clinical Laboratory Weifang People's Hospital 151 Guangwen Street Weifang 261041 Shandong China

E-mail: lab_lwx@126.com 